International Journal of Mathematical Analysis

Vol. 9, 2015, no. 1, 39 - 60

HIKARI Ltd, www.m-hikari.com

http://dx.doi.org/10.12988/ijma.2015.411373

\title{
Rate of Convergence to Equilibria of the Lotka-Sharpe-McKendrick Model
}

\author{
Larbi Alaoui \\ International University of Rabat \\ 11100 Sala Aljadida, Morocco \\ Mohamed Khaladi \\ Semlalia Faculty of sciences \\ 40000 Marrakech, Morocco
}

Copyright (c) 2014 Larbi Alaoui and Mohamed Khaladi. This is an open access article distributed under the Creative Commons Attribution License, which permits unrestricted use, distribution, and reproduction in any medium, provided the original work is properly cited.

\begin{abstract}
For the Lotka-Sharpe-McKendrick demographic model the rate of convergence to the persistent age distribution is estimated in terms of the age-dependent mortality and fertility. In an abstract setting the problems amounts to estimating the second largest spectral bound of a linear operator. The convergence rate is indeed characterized as the difference between the first and second spectral bounds and related estimation results are given.
\end{abstract}

Keywords: age structure, persistent solution, convergence to equilibrium, spectral bound

\section{Introduction}

In continuous time dynamical systems the convergence to equilibrium in the neighbourhood of an attracting stationary point can be estimated by the spectral bound $s$ of the Jacobian at that stationary point. The spectral bound is 
the largest real part of any eigenvalue. In many cases the problem of estimating $s$ amounts to finding a bound on the largest eigenvalue of some matrix or the largest root of some polynomial. This task is a classical problem in matrix theory.

Systems which describe demographic models are usually homogeneous, if not even linear. In homogeneous systems the role of stationary solutions is assumed by exponential solutions [18]. In the demographic context these represent exponentially growing populations with stable age structure. Then the exponent $\hat{\lambda}$ of the exponential solution is itself an eigenvalue of the Jacobian. In this case the condition for linear stability (in continuous time) requires that the differences $\operatorname{Re}(\lambda)-\hat{\lambda}$ are negative, where $\lambda$ varies over the eigenvalues other than $\hat{\lambda}$ (multiplicities counted). Then the rate of convergence is given by the largest difference $\operatorname{Re}(\lambda)-\hat{\lambda}$.

Thus the problem of estimating the rate of convergence to an exponential solution amounts to finding a bound for the "second largest" eigenvalue of the Jacobian, i.e., the eigenvalue with second largest real part. Apparently there are no tools for this problem in the general case, in particular for problems in infinite dimensions.

Similar problems arise in discrete time systems. Whereas in the standard situation of an attracting fixed point the spectral radius of the Jacobian governs the rate of convergence to equilibrium, in homogeneous systems the second largest eigenvalue (in modulus) determines the rate of convergence to the persistent solution.

The question addressed is most important in population dynamics where the exponential solutions correspond to persistent population distributions with exponential growth of the population. Most of the classical models are linear and the dynamical systems preserve positivity. Then the problem amounts to estimating the "second" eigenvalue of a positive operator, where "second" means "second in modulus" in the discrete time case and "second in real part" in the continuous time case.

The problem of estimating the second eigenvalue of a positive operator has been treated within the framework of Hilbert's projective metric in several classical papers. The main results of the classical theory are Birkhoff's and E. Hopf's inequalities [11], [9], [19] and the inequality of Bauer, Deutsch and Stoer [10], see also [16], [25]. Unfortunately, these inequalities give rather weak results when applied to demographic models.

In the classical demographic literature there are many attempts to estimate the exponent of demographic growth in terms of the birth and death rates (estimating Lotka's r), see, e.g., [13], [20]. Apart from rather evident observations (the exponent is an increasing function of the birth rate and a decreasing function of the death rate) it is difficult to estimate this quantity in case of nonuniform changes of these functions. It seems even more difficult 
to find estimates for the second eigenvalue.

A fundamental obstacle in the treatment of this problem is the lack of insight into its true physical nature. It is not intuitively evident what properties of the birth and death rates lead to quick equilibration after perturbations.

Here we present an analysis of the problem which yields some rigorous, though not exhausting, results. In Section 2, we consider the problem of estimating the second largest eigenvalue for the famous Lotka-Sharpe-McKendrick demographic model which reads $([28],[24])$

$$
\begin{array}{r}
\frac{\partial u}{\partial t}+\frac{\partial u}{\partial a}=-\mu(a) u \\
u(t, 0)=\int_{0}^{\infty} b(a) u(t, a) d a
\end{array}
$$

(See, e.g., [29] and [1-2] for the general theory and mathematical background, and [20] for the significance in demography. The first equation in (1) is also called Lotka-Von Foerster equation). We give multiple estimations of the speed of convergence of solutions towards the steady state based on the properties of the functions $\mu$ and $b$. This is done by establishing estimates for the associated second eigenvalue $s$ and also lower and upper bounds for $s$ relative to the first leading eigenvalue $\hat{\lambda}$ that defines the exponent of demographic growth.

In Section 3 we consider the Hopf's inequality and we explore its possible applications to the problem related to the rate of convergence to demographic

equilibrium. The Hopf's inequality allows us for the case of the finite dimensional space $X=\mathbb{R}^{n}$ to give good result for estimates of the second value relative to the leading eigenvalue.

In Section 4, the case of Leslie model is treated. This model is considered as the analogue of the Lotka-Sharpe-McKendrick model for the discrete time case [22]. We also get a similar result as in the continuous case with respect to the root of the associated characteristic polynomial.

Our results are also motivated by discussions based on illustrative examples.

\section{The classical demographic model}

The persistent solution of Lotka-Sharpe-McKendrick system (1) has the form $([29],[1-2])$

$$
u(t, a)=e^{\hat{\lambda} t} \bar{u}(a)
$$

where $\hat{\lambda}$ is the exponent of demographic growth and $\bar{u}$ is the persistent age distribution. The exponent $\hat{\lambda}$ is the leading root ([29], [1-2]) of the characteristic equation

$$
\int_{0}^{\infty} b(a) e^{-\int_{0}^{a} \mu(s) d s} e^{-\lambda a} d a=1
$$


and

$$
\bar{u}(a)=e^{-\int_{0}^{a} \mu(s) d s-\lambda a} .
$$

It is well known that equation (3) has a single (simple) real root $\hat{\lambda}$. Any other root $\lambda$ is necessarily complex and satisfies $\operatorname{Re}(\lambda)<\hat{\lambda}$. Let $s_{1}$ be the maximal real part among the roots apart from $\hat{\lambda}$.

The persistent age distribution $\bar{u}$ depends on the age dependent mortality $\mu(a)$ and on the exponent $\hat{\lambda}$, which in turn depends on $\mu(a)$ and $b(a)$. For given $\mu$, the exponent $\hat{\lambda}$ can assume a wide range of values depending on the function $b(a)$. E.g., for $\mu \equiv$ const., the exponent can be any number $\hat{\lambda}>-\mu$.

For positive values of $\hat{\lambda}$ the function $\bar{u}(a)$ is decreasing, but for negative $\hat{\lambda}$ (corresponding to a high mortality - low fertility situation) the function $\bar{u}(a)$ typically becomes "onion-shaped".

The exponent $\hat{\lambda}$ does not depend on the functions $\mu(a)$ and $b(a)$ separately but only on the kernel

$$
k(a)=p(a) b(a)=b(a) e^{-\int_{0}^{a} \mu(s) d s}
$$

where $p(a)=\exp \left\{-\int_{0}^{a} \mu(s) d s\right\}$ is the survival function. Then equation (3) reads

$$
\int_{0}^{\infty} k(a) e^{-\lambda a} d a=1
$$

Although mathematically the numbers $\hat{\lambda}$ and $s_{1}$ depend only on the function $k$, from a demographer's point of view the separate effects of the functions $b(a)$ and $\mu(a)$ are of interest.

Throughout the paper we assume that $b \geq 0$ and that $b$ is positive on some interval. The extreme case of fast equilibration in the evolution of (1) occurs when there are no other characteristic roots, i.e., if the spectral bound is formally $s_{1}=-\infty$. An extremal example is easily found: Choose $b(a) \equiv b$ and $\mu(a) \equiv \mu$ as positive constants. Then $k(a)=b e^{-\mu a}$ and $\hat{\lambda}=b-\mu, s_{1}=-\infty$. This example shows that in general the integral (6) does not exist for $\lambda<<0$. The following trivial observation is essential. If the kernel $k(a)$ is replaced by $k(a) e^{-\kappa a}$, then any eigenvalue $\lambda$ is replaced by $\lambda-\kappa$. In particular, $s_{1}-\hat{\lambda}$ stays the same.

Thus, we have found an extremal two-parameter family. However, from the fact that $k$ is an exponential function we cannot conclude that $b$ and $\mu$ are constants. Indeed, for any $\varphi(a)>0, \tilde{b}(a)=b \varphi(a), \tilde{\mu}(a)=\mu(a)+\varphi^{\prime}(a) / \varphi(a)$, we obtain the same kernel $k$. Therefore it seems wise to look for properties of $k$ first and then return to $b$ and $\mu$. First we prove a lemma which is basic for all subsequent estimates.

In the following we assume $k(a) \geq 0, k(a) \not \equiv 0$. 
Lemma 2.1 Let $k \in L^{1}(0, \infty)$, $k$ absolutely continuous and nonincreasing. Let $\gamma \in L_{\text {loc }}^{1}(0, \infty)$ be such that the product $k \gamma$ satisfies $k \gamma \in L^{1}(0, \infty)$. Let

$$
\gamma_{1}(a)=\int_{0}^{a} \gamma(s) d s, \quad \gamma_{2}(a):=\int_{0}^{a} \gamma_{1}(s) d s .
$$

i) Let $\gamma_{1}(a)>0$ a.e. in $(0, \infty)$ and uniformly bounded. Then

$$
\int_{0}^{\infty} k(a) \gamma(a) d a \geq 0
$$

and the inequality is strict.

ii) Assume that $k$ is convex. Let $\gamma_{1} \in L_{\text {loc }}^{1}(0, \infty), \gamma_{2}(a)>0$ a.e. in $(0, \infty)$. Let $\gamma_{1}$ and $\gamma_{2}$ be both uniformly bounded. Then (7) holds.

Proof. i) For $T>0$ we have

$$
\int_{0}^{T} k(a) \gamma(a) d a=\left[k(a) \gamma_{1}(a)\right]_{0}^{T}-\int_{0}^{T} k^{\prime}(a) \gamma_{1}(a) d a .
$$

We read this equation from right to left, let $T \rightarrow \infty$, and use $k(T) \rightarrow 0$. We find that $k^{\prime} \gamma_{1} \in L^{1}(0, \infty)$ and

$$
\int_{0}^{\infty} k(a) \gamma(a) d a=-\int_{0}^{\infty} k^{\prime}(a) \gamma_{1}(a) d a=\int_{0}^{\infty}\left|k^{\prime}(a)\right| \gamma_{1}(a) d a .
$$

Thus the integral (7) is nonnegative. It is zero only for $k^{\prime} \equiv 0$ which is excluded by the hypothesis.

ii) Consider, for $h>0$,

$$
k_{h}(a)=\frac{1}{h} \int_{a}^{a+h} k(s) d s \leq k(a)
$$

We know that $k_{h} \rightarrow k$ a.e. as $h \rightarrow 0$. The derivative is given by

$$
k_{h}^{\prime}(a)=\frac{k(a+h)-k(a)}{h} .
$$

Since $k$ is nonincreasing, we have $k_{h}^{\prime} \leq 0$. As before, we can write

$$
\int_{0}^{\infty} k_{h}(a) \gamma(a) d a=-\int_{0}^{\infty} k_{h}^{\prime}(a) \gamma_{1}(a) d a .
$$

In view of the convexity, the function $\left(-k_{h}^{\prime}\right)$ has the same properties as the function $k$ in i). Therefore, the quantity (9) is nonnegative. It is equal to zero only if $k_{h}^{\prime} \equiv 0$. In that case $k_{h} \equiv 0$, that is $k \equiv 0$ which is excluded. Thus the quantity (9) is even positive. Now let $h \rightarrow 0$ to obtain (7).

The following theorem gives a first estimate for the real part of the "second eigenvalue" $s_{1}$. This is an absolute bound, not relative to $\hat{\lambda}$. 
Theorem 2.2 Let $k: \mathbb{R}_{+} \rightarrow \mathbb{R}_{+}$be absolutely continuous and $k \not \equiv 0$. Then $s_{1}$ satisfies

$$
s_{1} \leq \sup _{a>0} \frac{k^{\prime}(a)}{k(a)} .
$$

Proof. Let $\lambda=\alpha+i \beta, \beta \neq 0$, be a root of (6). From (6) it follows that $\alpha<\hat{\lambda}$. Then

$$
\int_{0}^{\infty} k(a) e^{-\alpha a} \cos (\beta a) d a=1, \quad \int_{0}^{\infty} k(a) e^{-\alpha a} \sin (\beta a) d a=0 .
$$

Suppose $\alpha>\sup _{a>0} k^{\prime}(a) / k(a)$. Then $k(a) e^{-\alpha a}$ is nonincreasing. We use Lemma 2.1 , i) with $\gamma(a)=\sin \beta a$. The hypothesis is satisfied in view of

$$
\sin \beta a=\frac{2}{\beta} \frac{d}{d a} \sin ^{2} \frac{\beta a}{2} .
$$

Then

$$
\int_{0}^{\infty} k(a) e^{-\alpha a} \sin (\beta a) d a>0
$$

which contradicts (11).

Theorem 2.2 can be applied to $k(a) e^{-\hat{\lambda} a}$ instead of $k(a)$. Then one gets

$$
s_{1}-\hat{\lambda} \leq \sup _{a>0} \frac{\left(k(a) e^{-\hat{\lambda} a}\right)^{\prime}}{k(a) e^{-\hat{\lambda} a}} .
$$

However, this is only a reformulation of (10).

Corollary 2.3 Let $k$ be absolutely continuous. Assume there is a number $l \leq \hat{\lambda}$ such that the function $k(a) e^{-l a}$ is nonincreasing. Then the inequality

$$
s_{1} \leq l
$$

holds and thus $\hat{\lambda}-s_{1} \geq \hat{\lambda}-l$.

Proof. We apply Theorem 2.2 to the function $k_{1}(a)=k(a) e^{-l a}$ and get

$$
0 \geq \sup _{a>0}\left(k_{1}^{\prime}(a) / k_{1}(a)\right)=\sup _{a>0}\left(k^{\prime}(a) / k(a)\right)-l \geq s_{1}-l .
$$

Proposition 2.4 Assume that the derivative $k^{\prime}$ is absolutely continuous. Let $a k(a) \rightarrow 0$ and $a k^{\prime}(a) \rightarrow 0$ as $a \rightarrow \infty$. Let the function $\log k$ be convex. Then $s_{1}<0$.

Proof. Let $\lambda=\alpha+i \beta$, with $\alpha>0, \beta>0$, be a root of (6). Then

$$
\int_{0}^{\infty} k(a) e^{-\alpha a} \sin (\beta a) d a=\frac{1}{\beta} \int_{0}^{\infty}\left(a-\frac{\sin \beta a}{\beta}\right) e^{-\alpha a}\left[\alpha^{2} k(a)-2 \alpha k^{\prime}(a)+k^{\prime \prime}(a)\right] d a .
$$


In view of $k(a) \geq 0$ and the logarithmic convexity the expression in square brackets is a definite quadratic form in $\alpha$, thus nonnegative. Hence

$$
\int_{0}^{\infty} k(a) e^{-\alpha a} \sin (\beta a) d a \geq 0
$$

Suppose the inequality (15) is not strict. Then $\alpha^{2} k(a)-2 \alpha k^{\prime}(a)+k^{\prime \prime}(a) \equiv 0$, $k(a)=k(0) e^{\alpha a}$ which contradicts $\beta>0$. Thus the inequality (15) is strict. This again contradicts (11). for $\hat{\lambda}$.

The next corollary gives a lower bound for $\hat{\lambda}-s_{1}$ in terms of a lower bound

Corollary 2.5 Let $k$ be absolutely continuous. Assume there exists a number $l \leq \hat{\lambda}$ such that the function $k(a) e^{-l a}$ is nonincreasing. Let $A>0$ be arbitrary. Then

$$
\hat{\lambda}-s_{1} \geq \frac{1}{A} \log \int_{0}^{A} e^{-l a} k(a) d a .
$$

Proof. Evidently

$$
\int_{0}^{A} e^{-l a} k(a) d a=\int_{0}^{A} e^{(\hat{\lambda}-l) a} e^{-\hat{\lambda} a} k(a) d a \leq e^{(\hat{\lambda}-l) A} .
$$

Now take logarithms and use Corollary 2.3 to conclude the result.

Remark 2.6 i) Since $l<\hat{\lambda}$, we have $\int_{0}^{\infty} k(a) e^{-l a} d a>1$. If in (16) the number $A$ runs from 0 to $\infty$ then the right hand side runs from negative values to positive values and then decreases to 0 . Of course (16) is meaningful only if the right hand side is positive.

ii) From (17) it is obvious that Corollary 2.3 is stronger than Corollary 2.5 in case $\hat{\lambda}$ is known.

In realistic models, the function $k(a)$ will decrease for large a due to increasing mortality $\mu$ and decreasing fertility $b$, and it will vanish near $a=0$ because very young individuals have no offspring. For intermediate ages, the function $k(a)$, even in realistic models, may show rather arbitrary behavior, because the contributions of $b(a)$ and $\mu(a)$ may act in an antagonistic fashion. Therefore we consider the case where $k$ can have arbitrary behavior on a finite interval but satisfies assumptions as before for large a.

As before we state some preliminary results before giving estimates for $s_{1}-\hat{\lambda}$.

Lemma 2.7 Suppose $k$ is nonincreasing and positive, and $k$ is differentiable a.e.

i) Assume that $k^{\prime} / k$ is nondecreasing. Then $k^{\prime}$ is nondecreasing. 
ii) Suppose, in addition, that, for some $\alpha \in \mathbb{R}$, the function $e^{\alpha a} k(a)$ is nonincreasing. Then the function $\left(e^{\alpha a} k(a)\right)^{\prime}$ is nondecreasing.

Proof. We are interested in $k^{\prime}$ being nondecreasing. Suppose then that $k^{\prime} / k$ be nondecreasing. For each $a>b$, we have

$$
\frac{k^{\prime}(a)}{k(a)} \geq \frac{k^{\prime}(b)}{k(b)}
$$

which implies that

$$
\frac{k^{\prime}(a)}{k^{\prime}(b)} \leq \frac{k(a)}{k(b)}
$$

So, $k^{\prime}(a) / k^{\prime}(b) \leq 1$, which yields $k^{\prime}(a) \geq k^{\prime}(b)$.

The condition that $k^{\prime} / k$ be nondecreasing is very restrictive, but it is insensitive to multiplication of $k$ by any exponential. That is, let $\alpha \in \mathbb{R}$ be such that $e^{\alpha a} k(a)$ is nonincreasing. Then if $k^{\prime} / k$ is nondecreasing, the same is true for $\tilde{k}^{\prime} / \tilde{k}$, where $\tilde{k}(a)=e^{\alpha a} k(a)$.

Proposition 2.8 Let $k>0$ be absolutely continuous. Denote

$$
C(\alpha, \beta)=\int_{0}^{\infty} k(a) e^{-\alpha a} \cos (\beta a) d a, \quad S(\alpha, \beta)=\int_{0}^{\infty} k(a) e^{-\alpha a} \sin (\beta a) d a .
$$

Assume there exists $M>0$ such that the function $k(a) e^{M a}$ is nonincreasing and in $L^{1}(0, \infty)$. Assume furthermore that $k^{\prime} / k$ is nondecreasing (or $M>2 \pi$, respectively). Then for each $0 \geq \alpha \geq-M(0 \geq \alpha \geq 2 \pi-M$, respectively) and $\beta>0$ we have $C(\alpha, \beta)>0$ and $(\alpha, \beta)>0$.

Proof. Let $0 \geq \alpha \geq-M$. Let $\tilde{k}(a)=e^{-\alpha a} k(a)$. Then $\tilde{k}$ is nonincreasing and in $L^{1}(0, \infty)$, and $\tilde{k}^{\prime}$ is nondecreasing by Lemma 6 . Thus $\tilde{k}$ satisfies the hypothesis of Lemma 2.1. Hence $C(\alpha, \beta)>0, S(\alpha, \beta)>0$. On the other hand, if $M>2 \pi$, then for $2 \pi-M \leq \alpha \leq 0$, the functions $\gamma(a)=e^{-2 \pi a} \cos \beta a$ (resp. $\left.\gamma(a)=e^{-2 \pi a} \sin \beta a\right)$ and $k_{2}(a)=k(a) e^{M a} e^{-(\alpha+M-2 \pi) a}$ satisfy the conditions of Lemma 2.1, i), which yields $C(\alpha, \beta)=\int_{0}^{\infty} k_{2}(a) \gamma(a) d a>0$. (resp. $S(\alpha, \beta)>$ $0) . \diamond$ From now on, we choose $c>0$ and we write,

$$
k(a)= \begin{cases}k_{1}(a) & 0 j a j c \\ k_{2}(a-l) & \text { a } \mathrm{l} l\end{cases}
$$

where $k_{1}, k_{2}$ are nonnegative, $k_{2}$ nonincreasing. Then the characteristic equation reads, with $\lambda=\alpha+i \beta$,

$$
\int_{0}^{c} k_{1}(a) e^{-\alpha a} \cos (\beta a) d a+e^{-\alpha l} C_{2}(\alpha, \beta) \cos (\beta c)-e^{-\alpha c} S_{2}(\alpha, \beta) \sin (\beta c)=1
$$




$$
\int_{0}^{c} k_{1}(a) e^{-\alpha a} \sin (\beta a) d a+e^{-\alpha c} C_{2}(\alpha, \beta) \sin (\beta c)+e^{-\alpha c} S_{2}(\alpha, \beta) \cos (\beta c)=0
$$

where $C_{2}(\alpha, \beta), S_{2}(\alpha, \beta)$ are the functions defined in the above proposition for $k=k_{2}$.

Theorem 2.9 Assume that $\int_{0}^{\infty} k(a) d a=1$, and $k_{2} \not \equiv 0$ nonincreasing. Let

$$
\kappa=\inf _{a>0} \frac{\left|k_{2}^{\prime}(a)\right|}{k_{2}(a)}
$$

and

$$
\begin{gathered}
\eta=-\min \left[\kappa,-\frac{1}{c} \log \left(\frac{2 c}{\pi} k_{2}(0)+\int_{0}^{c} k_{1}(a) d a\right)\right] . \\
\hat{\eta}=-\min \left[\kappa-2 \pi,-\frac{1}{c} \log \left(\frac{2 c}{\pi} k_{2}(0)+\int_{0}^{c} k_{1}(a) d a\right)\right] .
\end{gathered}
$$

Assume furthermore that $k_{2}^{\prime} / k_{2}$ is nondecreasing (resp. $\kappa>2 \pi$ ). Then we have

$$
\hat{\lambda}=0 \quad \text { and } s_{1} \leq \eta \quad\left(\text { resp. } \quad s_{1} \leq \hat{\eta}\right)
$$

Proof. From the assumptions, it is immediate that $\hat{\lambda}=0$ is a root and no other real root can be found. So $s_{1} \leq 0$. Note that the estimate with $\eta$ is of interest only in the case

$$
\kappa>0 \text { and } \frac{2 l}{\pi} k_{2}(0)+\int_{0}^{l} k_{1}(a) d a<1,
$$

and it is obvious otherwise.

We will assume this hold. We show that the characteristic equation has no root $\lambda$ such that $\eta \leq \alpha=\operatorname{Re}(\lambda) \leq 0$ (resp. $\hat{\eta} \leq \alpha \leq 0$ ) and $\beta=\operatorname{Im} \lambda>0$.

Fix $\alpha$ such that $\eta \leq \alpha<0$ (resp. $\hat{\eta} \leq \alpha \leq 0$ ).

The fact that $\alpha>-\kappa$ implies that $k_{2}(a) e^{-\alpha a}$ is nonincreasing, $k_{2}(a) e^{-\alpha a}=$ $k_{2}(a) e^{\kappa a} e^{-(\kappa+\alpha) a}$ gives $k_{2}(a) e^{-\alpha a} \leq c e^{-(\kappa+\alpha) a} \in L^{1}(0,+\infty)$.

We also assumed that $k_{2}^{\prime} / k_{2}$ is nondecreasing (resp. $\kappa>2 \pi$ ). Therefore the conditions of Proposition 2.8 are verified, which implies that

$C_{2}(\alpha, \beta)>0$, and $S_{2}(\alpha, \beta)>0$ for $\alpha \geq-\kappa($ resp. $\alpha \geq 2 \pi-\kappa)$ and $\beta>0$

Coming back to the system of equations associated with the characteristic equation, we can determine $\cos (\beta l), \sin (\beta l)$ in terms of $C_{2}(\alpha, \beta)$, and $S_{2}(\alpha, \beta)$. Abbreviating these two latter functions to $C_{2}$ and $S_{2}$ respectively, we get

$$
\begin{aligned}
& \cos (\beta l)= \\
& \quad \frac{e^{\alpha l}}{C_{2}^{2}+S_{2}^{2}}\left[C_{2}\left(1-\int_{0}^{l} k_{1}(a) e^{-\alpha a} \cos (\beta a) d a\right)-S_{2} \int_{0}^{l} k_{1}(a) e^{-\alpha a} \sin (\beta a) d a\right]
\end{aligned}
$$




$$
\begin{aligned}
& \sin (\beta l)= \\
& \quad \frac{-e^{\alpha l}}{C_{2}^{2}+S_{2}^{2}}\left[C_{2} \int_{0}^{l} k_{1}(a) e^{-\alpha a} \sin (\beta a) d a+S_{2}\left(1-\int_{0}^{l} k_{1}(a) e^{-\alpha a} \cos (\beta a) d a\right)\right]
\end{aligned}
$$

Suppose now that $\alpha \geq(1 / l) \log \left(\int_{0}^{l} k_{1}(a) d a\right)$. Then if $\lambda=\alpha+i \beta$ is a root of the characteristic equation for some $\beta>0$, we claim that $\beta l \geq \pi$. In fact, let us assume for a moment that it is not so, that is $\beta l<\pi$. In this case we have

$$
\int_{0}^{l} k_{1}(a) e^{-\alpha a} \sin (\beta a) d a>0
$$

and

$$
1-\int_{0}^{l} k_{1}(a) e^{-\alpha a} \cos (\beta a) d a \geq 0,
$$

The latter inequality can be derived as follows

$$
\int_{0}^{l} k_{1}(a) e^{-\alpha a} \cos (\beta a) d a \leq \int_{0}^{l} k_{1}(a) e^{-\alpha l} d a=e^{-\alpha l} \int_{0}^{l} k_{1}(a) d a \leq 1
$$

by the assumption made on $\alpha$.

So, assuming $\beta l<\pi$, we conclude from the expression of $\sin (\beta l)$ that $\sin (\beta l)<$ 0 , which yields a contradiction. Therefore, we have $\beta l \geq \pi$. The claim is proved.

Suppose now that $-\kappa<\alpha \leq 0$ (resp. $2 \pi-\kappa<\alpha \leq 0)$ and

$$
|\alpha|<-\frac{1}{l} \log \left(\int_{0}^{l} k_{1}(a) d a\right)
$$

This implies both that $k_{2}(a) e^{-\alpha a}$ is decreasing and $\beta l>\pi$.

Coming back to the real part of the characteristic equation, we are led to

$$
\int_{0}^{l} k_{1}(a) e^{-\alpha a} \cos (\beta a) d a+\int_{0}^{\infty} k_{2}(a) e^{-\alpha(a+l)} \cos (\beta(a+l)) d a=1
$$

from which, using the second mean value theorem to estimate

$$
\int_{0}^{\infty} k_{2}(a) e^{-\alpha(a+l)} \cos (\beta(a+l)) d a, \quad \text { by } \quad k_{2}(0) e^{-\alpha l} \frac{2}{\beta}
$$

we arrive at

$$
1 \leq e^{-\alpha l}\left(\frac{2}{\beta} k_{2}(0)+\int_{0}^{l} k_{1}(a) d a\right)
$$


which leads readily to

$$
\alpha \leq \frac{1}{l} \log \left(\frac{2 l}{\pi} k_{2}(0)+\int_{0}^{l} k_{1}(a) d a\right) .
$$

That is

$$
|\alpha| \geq-\frac{1}{l} \log \left(\frac{2 l}{\pi} k_{2}(0)+\int_{0}^{l} k_{1}(a) d a\right) .
$$

Combining both inequalities and noting that

$$
-\frac{1}{l} \log \left(\frac{2 l}{\pi} k_{2}(0)+\int_{0}^{l} k_{1}(a) d a\right) \leq-\frac{1}{l} \log \left(\int_{0}^{l} k_{1}(a) d a\right)
$$

we arrive at the conclusion that

$$
|\alpha| \geq-\eta \quad \text { (resp. }|\alpha| \geq-\hat{\eta}) \text {. }
$$

in all cases, which yields the desired inequality for $s_{1}$.

Remark 2.10 As $l \rightarrow 0$ the estimate $\eta$ tends to $-\kappa$, the value given in the case $l=0$ in Theorem 2.9 .

If in the other hand, $l \rightarrow+\infty$, in such a way that

$$
\begin{gathered}
k_{1} \rightarrow k \quad(\text { for some function } \mathrm{k}) \\
k_{2}(a)=A e^{-\kappa a} \\
\int_{0}^{\infty} k_{2}(a) d a=A / \kappa \rightarrow 0
\end{gathered}
$$

then the estimate tends to zero. The estimate $\eta$ is non trivial if

$$
\int_{0}^{l} k_{1}(a) d a+\frac{2 l}{\pi} k_{2}(0)<1
$$

or

$$
\frac{2 l}{\pi} k_{2}(0)<1-\int_{0}^{l} k_{1}(a) d a=\int_{0}^{\infty} k_{2}(a) d a .
$$

So, the condition for non triviality is that

$$
\frac{k_{2}(0)}{\int_{0}^{+\infty} k_{2}(a) d a} l<\frac{\pi}{2}
$$

- In terms of $l, k_{2}$ being fixed, it can be achieved by letting $l>0$ small enough. 
- In terms of $\kappa$, we have

$$
\frac{k_{2}^{\prime}(a)}{k_{2}(a)} \leq-\kappa \Longrightarrow k_{2}(a) e^{\kappa a} \text { nonincreasing. }
$$

So,

$$
k_{2}(a) \leq k_{2}(0) e^{\kappa a} \Longrightarrow \int_{0}^{\infty} k_{2}(a) d a \leq \frac{1}{\mu} k_{2}(0) .
$$

Then the condition of non triviality implies

$$
\kappa l<\frac{\pi}{2}
$$

Consider now the case when

$$
\int_{0}^{\infty} k(a) d a \neq 1
$$

The largest root $\hat{\lambda}$ is then $\neq 0$.

Denote

$$
\tilde{k}(a)=e^{-\hat{\lambda} a} k(a)
$$

The above remark leads to a method giving a non trivial estimate of $\hat{\lambda}-s_{1}$. The method consists in looking for $l \geq 0$ so that $\tilde{k}(a)$ can be written as follows

$$
\begin{aligned}
& \tilde{k}(a)=\tilde{k}_{1}(a) \text { on }(0, l) \\
& \tilde{k}(a)=\tilde{k}_{2}(a-l) \text { on }(l,+\infty)
\end{aligned}
$$

where $\tilde{k}_{1}$ (resp. $\left.\tilde{k}_{2}\right)$ verify the same properties as $k_{1}$ (resp. $k_{2}$ ).

For such a decomposition to be possible, it is necessary that $k(a) e^{-\hat{\lambda} a}$ be nonincreasing for $a>l$, and $k^{\prime} / k$ nondecreasing on the same interval.

Let us examine this condition. $k(a) e^{-\hat{\lambda} a}$ being nonincreasing is equivalent to $k^{\prime}(a)-\hat{\lambda} k(a) \leq 0$ for a large enough, that is

$$
\hat{\lambda} \geq \frac{k^{\prime}(a)}{k(a)}
$$

This means that

$$
\hat{\lambda} \geq \lim _{a \rightarrow+\infty} \frac{k^{\prime}(a)}{k(a)} .
$$

To be more precise, we will state the following result. 
Corollary 2.11 Let us assume that $\int_{0}^{\infty} k(a) d a \geq 1$ and

$$
\kappa=\inf _{a>0} \frac{\left|k_{2}^{\prime}(a)\right|}{k_{2}(a)}>0
$$

Let us assume moreover that for $a>l, k^{\prime} / k$ is nondecreasing (resp. $\kappa>2 \pi$ ), and

$$
\int_{0}^{l} e^{\kappa a} k(a) d a<1 \text { and } \frac{e^{\kappa l} k(l)}{1-\int_{0}^{l} e^{\kappa a} k(a) d a} l<\frac{\pi}{2} .
$$

Then we have

$$
\begin{aligned}
& s_{1}-\hat{\lambda} \leq-\min \left[\kappa, L:=-\frac{1}{l} \log \left(\frac{2 l}{\pi} e^{\kappa l} k(l)+\int_{0}^{l} e^{\kappa a} k(a) d a\right)\right] \\
& \left(\text { resp. } \quad s_{1}-\hat{\lambda} \leq-\min (\kappa-2 \pi, L)\right)
\end{aligned}
$$

\section{Proof.}

The function $\tilde{k}(a)=e^{-\hat{\lambda} a} k(a)$ satisfies the assumptions of Theorem 2.9. Then we have

$$
\begin{aligned}
& \tilde{s}_{1} \leq-\min \left[\tilde{\kappa}, \tilde{L}:=-\frac{1}{l} \log \left(\frac{2 l}{\pi} \tilde{k}_{2}(0)+\int_{0}^{l} \tilde{k}_{1}(a) d a\right)\right] \\
& \left(\operatorname{resp} . \tilde{s}_{1} \leq-\min [\tilde{\kappa}-2 \pi, \tilde{L}]\right)
\end{aligned}
$$

with

$$
\begin{gathered}
\tilde{s}_{1}=s_{1}-\hat{\lambda}, \quad \tilde{\kappa}=\kappa+\hat{\lambda} \\
\int_{0}^{l} \tilde{k}_{1}(a) d a=\int_{0}^{l} e^{-\hat{\lambda} a} k_{1}(a) d a<\int_{0}^{l} e^{\kappa a} k(a) d a \\
\tilde{k}_{2}(0)=\tilde{k}(l)=e^{-\hat{\lambda} l} k(l) \leq e^{\kappa l} k(l)
\end{gathered}
$$

which implies that

$$
-\frac{1}{l} \log \left(\frac{2 l}{\pi} \tilde{k}_{2}(0)+\int_{0}^{l} \tilde{k}_{1}(a) d a\right) \geq-\frac{1}{l} \log \left(\frac{2 l}{\pi} e^{\kappa l} k(l)+\int_{0}^{l} e^{\kappa a} k(a) d a\right)
$$

and then we have

$$
s_{1}-\hat{\lambda} \leq-\min [\kappa+\hat{\lambda},-L] \quad\left(\text { resp. } \quad s_{1}-\hat{\lambda} \leq-\min [\kappa-2 \pi+\hat{\lambda}, L]\right) .
$$

This, with the fact that $\hat{\lambda}>0$, gives the desired estimate. 
Example 2.12 We will give an example for which the result of Remark 2.10 applies and for which we will schow how the conditions of Remark 2.10 can be satisfied. Take

$$
k(a)=\frac{c}{1+a} e^{-\kappa a}
$$

where $c$ and $\kappa$ are positive numbers. In this case

$$
\begin{gathered}
\frac{k^{\prime}(a)}{k(a)}=-\kappa-\frac{1}{1+a} \text { and } \lim _{a \rightarrow+\infty} a k^{\prime}(a)=0 \\
\frac{k^{\prime}}{k} \text { is increasing and } \lim _{a \rightarrow+\infty} \frac{k^{\prime}(a)}{k(a)}=-\kappa .
\end{gathered}
$$

On the other hand

$$
\begin{gathered}
\int_{0}^{l} e^{\kappa a} k(a) d a=\int_{0}^{l} \frac{c}{1+a} d a=c \log (1+l) \\
\frac{e^{\kappa l} k(l)}{1-\int_{0}^{l} e^{\kappa a} k(a) d a} l=\frac{c /(1+l)}{1-c \log (1+l)} l
\end{gathered}
$$

So, $\int_{0}^{l} e^{\kappa a} k(a) d a$ and $\frac{e^{\kappa l} k(l)}{1-\int_{0}^{l} e^{\kappa a} k(a) d a} l$ both tend to zero as $l \rightarrow 0$. Then, for $l$ small enough, one has

$$
\int_{0}^{l} e^{\kappa a} k(a) d a<1 \quad \text { and } \frac{e^{\kappa l} k(l)}{1-\int_{0}^{l} e^{\kappa a} k(a) d a} l<\frac{\pi}{2} .
$$

Note that, in the present situation, we can fix $l$ and choose $c$ to have the conditions of Corollary satisfied.

Remark 2.13 i) For $\hat{\lambda}<0$, that is for $\int_{0}^{\infty} k(a) d a<1$, we introduce as in Corollary 2.3, a "threshold" $a_{\kappa}$ such that

$$
\int_{0}^{a_{\kappa}} e^{\kappa a} k(a) d a=\frac{1}{2}\left(1+\int_{0}^{\infty} e^{\kappa a} k(a) d a\right) .
$$

Then, because $\int_{0}^{a_{\kappa}} e^{\kappa a} k(a) d a \leq e^{(\kappa+\hat{\lambda}) a_{\kappa}}$, we have

$$
\frac{1}{2}\left(1+\int_{0}^{\infty} e^{\kappa a} k(a) d a\right) \leq e^{(\kappa+\hat{\lambda}) a_{\kappa}}
$$

This yields

$$
\kappa+\hat{\lambda} \geq \frac{1}{a_{\kappa}} \log \left(\frac{1}{2}+\frac{1}{2} \int_{0}^{\infty} e^{\kappa a} k(a) d a\right) .
$$


Finally we obtain the following estimate

$s_{1}-\hat{\lambda} \leq-\min \left[\frac{1}{a_{\kappa}} \log \left(\frac{1}{2}+\frac{1}{2} \int_{0}^{\infty} e^{\kappa a} k(a) d a\right),-\frac{1}{l} \log \left(\frac{2 l}{\pi} e^{\kappa l} k(l)+\int_{0}^{l} e^{\kappa a} k(a) d a\right)\right]$

ii) For the Lotka-Von Foerster equation, realistic hypotheses on $\mu$ and $b$ lead to $k$ in the following form

$k=0$ on $(0, b)$ where $b$ is the age of start of fecundity,

$k$ non decreasing on $(b, l), l$ is the age of start of fecundity,

$k$ decreasing on $(l, L)$,

$k=0$ on $(L,+\infty)$.

So, all the estimates above are still valid in this situation, if in the hypotheses we replace $+\infty$ by $L$.

\section{Hopf's inequality.}

In this section we give a short account on Hopf's inequality and related estimates and we explore possible applications to the problem in population dynamics studied here.

Let $X=\mathbb{R}^{n}$ be endowed with the partial order $\geq$ induced by the cone $K=\mathbb{R}_{+}^{n}$ with interior $\stackrel{\circ}{K}$. Of course, vectors in $K$ are nonnegative and those in $\stackrel{\circ}{K}$ have all components positive. For $x \in \mathbb{R}^{n}, p \in \stackrel{\circ}{K}$ define the lower and upper "quotients" and the oscillation by

$$
\begin{gathered}
m_{1}(x \mid p)=\sup \{\alpha: x \geq \alpha p\}, \quad m_{2}(x \mid p)=\inf \{\alpha: x \leq \alpha p\} \\
o s c(x \mid p)=m_{2}(x \mid p)-m_{1}(x \mid p)
\end{gathered}
$$

For $p, q \in \stackrel{\circ}{K}$ define

$$
\vartheta(q \mid p)=m_{2}(q \mid p) / m_{1}(q \mid p), \quad \theta(q \mid p)=\log \vartheta(q \mid p) .
$$

The function $\theta(q \mid p)$ defines Hilbert's projective metric on $\stackrel{\circ}{K}$. For operators $A: X \rightarrow X$ with $A K \subset K$ there is a function $\mathcal{N}(A)$ taking values in $[0,1]$ that measures the distance of $A$ from the set of rank 1 operators. The number $\mathcal{N}(A)$ can be computed from the matrix representation $A=\left(a_{j k}\right)$ as follows. First define

$$
\vartheta(A)=\max _{j, k, s, t} \frac{a_{j s} a_{k s}}{a_{j t} a_{k t}}
$$

with $\vartheta(A)=\infty$ if any of the $a_{i j}=0$. Then define

$$
\mathcal{N}(A)= \begin{cases}\frac{\vartheta(A)^{1 / 2}-1}{\vartheta(A)^{1 / 2}+1} & \text { if } \vartheta(A) \neq \infty \\ 1 & \text { if } \vartheta(A)=\infty\end{cases}
$$


Thus $\mathcal{N}(A)<1$ if and only if $A$ is a positive matrix (i.e. if $A K \subset \stackrel{\circ}{K}$ ).

With this definition the Hopf ineqality holds

$$
\theta(A q \mid A p) \leq N(A) \theta(q \mid p)
$$

Thus $A$ is a (weak) contraction on $\stackrel{\circ}{K}$ with respect to the metric $\theta$.

For complex vectors $x=u+i v \in \mathbb{C}^{n}$ and positive vectors $p$ define

$$
\operatorname{osc}(x \mid p)=\sup _{\varphi \in \mathbb{R}} \operatorname{osc}(u \cos \varphi+v \sin \varphi / p) .
$$

Then the following inequality holds.

$$
\operatorname{osc}(A x / A p) \leq N(A) \operatorname{osc}(x / p) .
$$

Notice that $o s c$ is not a metric. Let $\rho(A)$ be the Perron root, $p$ a Perron eigenvector of $A$ and let $x$ be any other eigenvector, $A p=\rho p, A x=\lambda_{2} x$. From (18) it follows immediately the estimation of the following result.

Proposition 3.1 We have

$$
\left|\lambda_{2}\right| \leq \mathcal{N}(A) \rho(A) s .
$$

Thus Hopf's inequality yields a bound for the quotient of the "second" eigenvalue and the Perron root in case $A$ is a positive matrix.

Next, for a fixed vector $p \in \stackrel{\circ}{K}$, and for $x \in \mathbb{C}^{n}$, define the conditional oscillation

$$
\mathcal{N}(A \mid p)=\sup \{\operatorname{osc}(A q \mid A p): q \in \stackrel{\circ}{K}, \operatorname{osc}(q \mid p)=1\} .
$$

Then the inequality of Bauer, Deutsch and Stoer [10] says

$$
\operatorname{osc}(A x \mid p) \leq \mathcal{N}(A \mid p) \operatorname{osc}(x \mid p) .
$$

Since $\mathcal{N}(A, p) \leq \mathcal{N}(A)$, this inequality leads to a better estimate for the second eigenvalue.

Proposition 3.2 If $p$ is the Perron eigenvector of $A$ then

$$
\left|\lambda_{2}\right| \leq \mathcal{N}(A) s .
$$

Also this quantity can be expressed in terms of the matrix elements. For $p=$ $\left(p_{j}\right)>0$ define the matrix $B=\left(b_{j k}\right), b_{j k}=p_{j}^{-1} a_{j k} p_{k}$. Then

$$
N(B \mid p)=\frac{1}{2} \max _{1 \leq i, j \leq n} \sum_{k=1}^{n}\left|b_{i k}-b_{j k}\right|
$$

which yields the estimate of the following result.

Theorem 3.3 We have

$$
\left|\lambda_{2}\right| \leq \frac{1}{2} \max _{1 \leq i, j \leq n} \sum_{k=1}^{n} p_{k}\left|\frac{a_{i k}}{p_{i}}-\frac{a_{j k}}{p_{j}}\right| s .
$$




\section{The discrete time model.}

The analogue of the Lotka-Sharpe-McKendrick model for discrete time is the so-called Leslie matrix model [22]. It is nothing else than the much older Lewis model [23] in matrix notation. Let $u=\left(u_{1}, \ldots, u_{n}\right)^{T}$ be the (column) vector of age classes. Introduce the transition matrix

$$
A=\left(\begin{array}{ccccc}
b_{1} & b_{2} & \ldots & \ldots & b_{n} \\
\nu_{1} & 0 & 0 & \ldots & 0 \\
0 & \nu_{2} & 0 & \ldots & 0 \\
\vdots & \ddots & \ddots & \ddots & \vdots \\
0 & \ldots & 0 & \nu_{n-1} & 0
\end{array}\right)
$$

with survival rates $\nu_{j}>0$ and birth rates $b_{j} \geq 0$. We assume that not all $b_{j}$ vanish. Then the population dynamics is described by the matrix iteration

$$
u^{t+1}=A u^{t}, \quad t=0,1,2, \ldots
$$

. The matrix is, up to a similarity transform, a Frobenius companion matrix. Hence its characteristic polynomial can be easily computed,

$$
P(\lambda)=b_{1} \lambda^{n-1}+b_{2} \nu_{1} \lambda^{n-2}+\cdots+b_{n-1} \nu_{1} \nu_{2} \cdots \nu_{n-1}-\lambda^{n} .
$$

Thus $P(\lambda)$ is a so-called Cauchy polynomial: the leading coefficient is -1 , all remaining coefficients are nonnegative and do not all vanish. The Perron root $\rho=\rho(A)$ is the only positive real root of $P$, the other roots do not exceed this root in modulus. The root $\rho$ can be bounded in terms of the coefficients $b_{j}$ and $p_{j}$ in various ways. The best bound is just $\rho$ itself.

There has been considerable interest in the set of all zeros of all Cauchy polynomials with normalization $\rho=1$ ([21], [16], [17]) but these results do not give information on $\lambda_{2}$ for a particular polynomial. The second largest root $\lambda_{2}$ is nonpositive real or complex. For large $n$ it can be located rather close to the positive real axis.

We observe that (19) cannot be directly applied to the matrix $A$ since $A$ has zero elements $(N(A)=1)$.

Then we observe a somewhat strange discrepancy. The optimal matrices in the context of Hopf's inequality are those with $\mathcal{N}(A)=0$, i.e. matrices of rank $1, A=\xi \eta^{T}$, where $\xi, \eta$ are positive vectors. On the other hand the optimal polynomial in the sense that $\lambda_{2}=0$ is $P(\lambda)=\rho \lambda^{n-1}-\lambda^{n}$, and its companion matrix (a cyclic matrix) is very far from the above. It correponds to the demographic model in which only the youngest age class reproduces. In this situation the other classes could be dropped rightaway. Also the bound (20) is not really useful. Thus it seems that looking at the discrete case in the way of matrices is the wrong approach. Indeed, the concept of matrix 
introduces the order $n$ of that matrix or the degree of the polynomial which is not innate to the demographic problem while the behavior of polynomials depends heavily on the degree.

Thus we start again from the demographic problem, this time more closely imitating the continuous case. We assume that there is an infinite number of age classes (all but finitely many empty) $u_{j}^{t}, j=1,2, \ldots$, and we write the evolution equation The second largest root $\lambda_{2}$ is nonpositive real or complex. For large $n$ it can be located rather close to the positive real axis.

$$
\left\{\begin{array}{l}
u_{1}^{t+1}=\sum_{j=1}^{\infty} b_{j} u_{j}^{t} \\
u_{j}^{t+1}=\nu_{j-1} u_{j-1}^{t} \quad j>1
\end{array}\right.
$$

Then the characteristic polynomial (whatever its degree may be) is obtained from

$$
\begin{gathered}
\left\{\begin{array}{l}
\lambda u_{1}=\sum_{j=1}^{\infty} b_{j} u_{j} \\
\lambda u_{j}=\nu_{j-1} u_{j-1} \quad j>1
\end{array}\right. \\
\lambda u_{1}=b_{1} u_{1}+b_{2} \nu_{1} \frac{1}{\lambda} u_{2}+b_{3} \nu_{2} \nu_{1} \frac{1}{\lambda^{2}} u_{3}+\cdots
\end{gathered}
$$

we get the characteristic polynomial in the form

$$
\lambda=k_{1}+k_{2} \frac{1}{\lambda}+k_{3} \frac{1}{\lambda^{2}}+\cdots
$$

with $k_{j}=b_{j} p_{j}$. Now the degree does not show up explicitly. Even more, for realistic parameters, the coefficients decrease for large $j$. We therefore get the following result which is more or less the same thing as (11).

Proposition 4.1 If $\lambda=r e^{i \varphi}$ is a root then

$$
\begin{aligned}
& 1=k_{1} \frac{1}{r} \cos \varphi+k_{2} \frac{1}{r^{2}} \cos 2 \varphi+k_{3} \frac{1}{r^{3}} \cos 3 \varphi+\cdots \\
& 0=k_{1} \frac{1}{r} \sin \varphi+k_{2} \frac{1}{r^{2}} \sin 2 \varphi+k_{3} \frac{1}{r^{3}} \sin 3 \varphi+\cdots
\end{aligned}
$$

\section{Conclusion}

In this paper we addressed the problem of determining the rate of convergence to demographic equilibrium for the famous Lotka-Sharpe-McKendrick model by characterizing it as the difference between the largest and the second largest spectral bounds. We established different associated results by giving accurate estimations of bounds of this difference. Also a connection of this problem to 
Hopf's inequality was introduced in order to explore the possible applications of this inequality for the determination of properties on the first and the second largest spectral bounds.

It is to be noticed that the methods proposed and the results obtained can be adapted for applicationto many other models from population dynamics. It is indeed to be noticed that the Lotka-Sharpe-McKendrick is used as a basis model for the mathematical modeling in multiple application domains of population dynamics (eg., [1-6], [7-8], [12], [14-15], [26-27], [29-31]). The models obtained for such applications are simply variations or extensions of this model where either new parameters are added with respect to the application in purpose or some perturbation factors are used. Furthermore the mathematical analysis tools generally used for the study of such models are generally based the same approaches and techniques used for the Lotka-Sharpe-McKendrick model. For most of these models the analysis of their solutions with the aim of yielding conditions under which qualitative results could be deduced (eg., property of asynchronous exponential growth, stability of steady states) has a attracted many researchers in the last two decades (eg., [1-7], [1-6], [7-8], [12], [14-15], [27], [29-31]).

We are confident that our results given above on the second and first largest spectral bounds will pave the way for better understanding of the dynamics behind such models by treating the question on how quickly the convergence to their corresponding equilibria is guaranteed.

Acknowledgements. This work is extracted from the dissertation [5] of the doctorate thesis that was supervised by Prof. K.P. Hadeler. The authors would like to thank Prof. Dr. Hadeler and also Prof. Dr. O. Arino for their valuable suggestions and comments.

\section{References}

[1] L. Alaoui, Nonlinear retarded differential equations and population dynamics via translation semigroups, Semigroup Forum Vol. 63 (2001) 330356.

[2] L. Alaoui, Age-dependent population dynamics and translation semigroups, Semigroup Forum 57 (1998). http://dx.doi.org/10.1007/pl00005973

[3] L. Alaoui, A cell cycle model and translation semigroups, Semigroup Forum 54 (1997). http://dx.doi.org/10.1007/bf02676597 
[4] L. Alaoui, Generators of Translation Semigroups and Asymptotic Behavior of the Sharpe-Lotka Model, Diff. and Int. Eq., Vol 9, n. 2, March (1996).

[5] L. Alaoui, Population dynamics and translation semigroups, dissertation, University of Tübingen (July 1995).

[6] L. Alaoui, O. Arino, Compactness and spectral properties for positive translation semigroups associated to models of populations dynamics, Differential and Integral Equations, 6, 1993, 459-480.

[7] O. Arino, E. Sanchez, and G. F. Webb. Necessary and sufficient conditions for asynchronous exponential growth in age structured cell populations with quiescence, J. Math. Anal. Appl., 215(2):499513, 1997. http://dx.doi.org/10.1006/jmaa.1997.5654

[8] P. Auger, R. Bravo de la Parra, J.-C. Poggiale, E. Snchez, and T. NguyenHuu5, Aggregation of Variables and Applications to Population Dynamics, In "Structured population models in biology and epidemiology," (P. Magal, S. Ruan (Eds.)) Springer-Verlag Berlin Heidelberg (2008). http://dx.doi.org/10.1007/978-3-540-78273-5_5

[9] F. L. Bauer, An elementary proof of the Hopf inequality for positive operators, Numer. Math. 7, 331-337 (1965). http://dx.doi.org/10.1007/bf01436527

[10] F. L. Bauer, E. Deutsch, J. Stoer, Abschätzungen für die Eigenwerte positiver linearer Operatoren, Lin. Algebra and its Appl. 2, 275-301 (1969). http://dx.doi.org/10.1016/0024-3795(69)90031-7

[11] G. Birkhoff, Extensions of Jentzsch's theorem, Trans. Amer. Math. Soc. 85, 219-227 (1957). http://dx.doi.org/10.1090/s0002-9947-1957-00870586

[12] M. Boulanouar, Asynchronous Exponential Growth of a Bacterial Population, Electronic Journal of Differential Equations, Vol. 2014 (2014), No. 06.

[13] A. J. Coale, The growth and structure of human papulations. A mathematical investigation, Princeton University Press, Princeton, N.J., (1972).

[14] A. L. Dawidowicz1 and A. Poskrobko, On asymptotic behaviour of the dynamical systems generated by von Foerster-Lasota equations, Control and Cybernetics, vol. 35 (2006) No. 4 
[15] O. Diekmann, H. J. A. M. Heijmans, and H. R. Thieme, On the stability of the cell size distribution, J. Math. Biol., 19:227248, 1984. http://dx.doi.org/10.1007/bf00277748

[16] K. P. Hadeler, Abschätzungen für den zweiten Eigenwert eines positiven Operators, AEQ. Math. Vol. 5, 227-235 (1970). http://dx.doi.org/10.1007/bf01818444

[17] K. P. Hadeler, G. Meinardus, On the roots of Cauchy polynomials, Linear Algebra and its Applications 38, 81-102 (1981). http://dx.doi.org/10.1016/0024-3795(81)90010-0

[18] K. P. Hadeler, Periodic solutions of homogeneous equations, J. Diff. Eq. 95, 183-202 (1992). http://dx.doi.org/10.1016/0022-0396(92)90049-s

[19] E. Hopf, An inequality for positive linear integral operators, J. Math. Mech. 12, 683-692 (1963).

[20] N. Keyfitz, Introduction to the mathematics of population with revisions, Addison-Wesley, Reading, Mass., (1977).

[21] S. Kirkland, On the spectrum of a Leslie matrix with a near-periodic fecundity pattern, Linear Algebra and its Applications, 178, 261-279 (1993). http://dx.doi.org/10.1016/0024-3795(93)90345-o

[22] P. H. Leslie, On the use of matrices in certain population mathematics, Biometrika 33 (1945), 183-212. http://dx.doi.org/10.1093/biomet/33.3.183

[23] E. G. Lewis, On the generation and growth of a population, Sankhya 6 (1942), 93-96.

[24] A.G. McKendrick, Applications of mathematics to medical problems, Proc. Edinb. Math. Soc. 44 (1926), pp. 98-130. http://dx.doi.org/10.1017/s0013091500034428

[25] A. M. Ostrowski, Positive matrices and functional analysis, in Recent Advances in Matrix Theory (Univ. of Wisconsin Press, Madison 1964) 81-101.

[26] B. Perthame, Transport equations arising in biology, in Frontiers in Mathematics, Frontiers in Mathematics, Birkhauser, 2006.

[27] B. Perthame, L. Ryzhik, Exponential decay for the fragmentation or cell-division equation, J. Differential Equations, 210 (2005), pp. 155177. http://dx.doi.org/10.1016/j.jde.2004.10.018 
[28] F.R. Sharpe, A.J. Lotka, A problem in age distributions, Phil. Mag. 21 (1911), pp. 435-438. http://dx.doi.org/10.1080/14786440408637050

[29] G. F. Webb, "Theory of nonlinear age-dependent population dynamics", M. Dekker, New York and Basel (1985).

[30] G. F. Webb, An operator-theoretic formulation of asynchronous exponential growth, Trans. Amer. Math. Soc., 303(2):751763, (1987). http://dx.doi.org/10.1090/s0002-9947-1987-0902796-7

[31] G.F. Webb, Population Models Structured by Age, Size, and Spatial Position In "Structured population models in biology and epidemiology," (P. Magal, S. Ruan (Eds.)) Springer-Verlag Berlin Heidelberg (2008). http://dx.doi.org/10.1007/978-3-540-78273-5_1

Received: December 4, 2014; Published: January 9, 2015 